\title{
Les champs littéraires africains, textes réunis et rassemblés par Romuald Fonkoua et Pierre Halen
}

\section{Maria Chiara Gnocchi}

\section{(2) OpenEdition}

1 Journals

\section{Édition électronique}

URL : https://journals.openedition.org/studifrancesi/39862

DOI : 10.4000/studifrancesi.39862

ISSN : 2427-5856

Éditeur

Rosenberg \& Sellier

\section{Édition imprimée}

Date de publication : 1 décembre 2004

Pagination : 414-416

ISSN : 0039-2944

\section{Référence électronique}

Maria Chiara Gnocchi, « Les champs littéraires africains, textes réunis et rassemblés par Romuald

Fonkoua et Pierre Halen », Studi Francesi [En ligne], 143 (XLVIII | II) | 2004, mis en ligne le 30 novembre 2015, consulté le 19 mai 2021. URL : http://journals.openedition.org/studifrancesi/39862 ; DOI : https://doi.org/10.4000/studifrancesi.39862

Ce document a été généré automatiquement le 19 mai 2021.

\section{(c)}

Studi Francesi è distribuita con Licenza Creative Commons Attribuzione - Non commerciale - Non opere derivate 4.0 Internazionale. 


\title{
Les champs littéraires africains, textes réunis et rassemblés par Romuald Fonkoua et Pierre Halen
}

\author{
Maria Chiara Gnocchi
}

\section{RÉFÉRENCE}

Les champs littéraires africains, textes réunis et rassemblés par Romuald fonKOUA et Pierre HALEN, avec la collaboration de Katharina STÄDTLER, Paris, Karthala, 2001, pp. 344.

1 La notion de champ littéraire, introduite par Pierre Bourdieu en 1984 et développée de façon plus complète dans le volume Les Règles de l'Art (Seuil, 1992) a été, depuis, largement exploitée et appliquée par les chercheurs, au niveau international. Initialement formulée pour rendre compte de la situation culturelle française (moderne et contemporaine), elle s'est révélée particulièrement utile pour l'étude des différentes réalités littéraires "marginales" (des manifestations artistiques des provinces françaises jusqu'à ce qu'on appelle la paralittérature). Les études rassemblées par Romuald Fonkoua et Pierre Halen sous le titre Les champs littéraires africains (issues d'un colloque international de l'Association pour l'Étude des Littératures africaines, APELA, qui eut lieu à Bruxelles en 1997) entendent précisément vérifier la pertinence et la portée d'une telle approche critique et méthodologique pour l'étude des littératures africaines modernes.

2 L'analyse d'un tel sujet présente une série de difficultés: non seulement différentes catégories de "littérature africaine" coexistent avec celle sur laquelle on se propose de travailler ici, mais les vingt dernières ont vu s'accentuer l'écart entre deux différents types de circuits de production, possédant chacun ses propres codes d'appréciation: les circuits locaux et les circuits internationaux. Une mise au point parait d'autant plus souhaitable que la critique s'est, elle aussi, considérablement développée et diversifiée, en termes d'objet mais aussi de méthode. Notons que l'approche dont les membres de 
l'Association pour l'Étude des Littératures africaines ont été invités à évaluer la pertinence ne saurait se résoudre aux seuls travaux de Bourdieu et de ses disciples les plus orthodoxes: il s'agit plutôt de la synthèse d'une série d'approches résumées, dans l'avant-propos au volume, par l'étiquette d'institutionnelles, et qui voient, entre autres, les efforts critiques de Jacques Dubois, de Jean-Marie Klinkenberg, de Paul Aron et de Paul Dirkx rejoindre, compléter, parfois même corriger, les thèses de Bourdieu. L'analyse institutionnelle des littératures africaines avait déjà été entamée, mais par des travaux portant sur les propriétés du discours plutôt que sur la circulation matérielle des biens culturels et symboliques. Les essais dont on rend compte ici se situent par contre pour la plupart "dans cette intervalle entre approche des représentations et analyse des structures institutionnelles. Leur portée est donc de marquer une étape sur la voie d'une appréhension globale des littératures africaines, envisagées à la fois dans les fonctions sociales qu'elles remplissent et dans le fonctionnement, non moins socialisé, de leur récente légitimité" (Avant-propos, p. 14).

3 Le volume s'ouvre par des "Questions de méthode" confiées à des spécialistes, non des littératures africaines, mais de l'analyse institutionnelle. Ainsi, Pascal Durand (Introduction à une sociologie des champs symboliques) expose-t-il la théorie du champ littéraire, tout en essayant de la concilier avec celle de l'institution de la littérature (sur le modèle proposé par Dubois). Son excellent condensé d'histoire sociale de la littérature (l'autonomisation et la professionnalisation progressive de la littérature, donc sa constitution en tant que champ doté de règles propres) sert précisément à marquer les écarts entre la situation française, ou en tout cas européenne, et la situation africaine.

De sa part, Paul Aron aborde Le fait littéraire francophone dans son ensemble, et essaie de définir les conditions d'existence spécifiques aux littératures francophones. Les questions inaugurales qu'il pose sont les suivantes: connaît-on tous les réseaux qui permettent à quelques rares auteurs "périphériques" d'accéder aux collections de poche françaises? A-t-on étudié (et même, a-t-on les moyens d'étudier) "les imbrications des milieux de la politique, de l'édition, de la coopération et de la presse qui font et défont les réputations littéraires des auteurs représentatifs de la francophonie"? (p. 43) Paul Aron invite les lecteurs à noter que "l'étude des littératures francophones implique un recours à des catégories étrangères à une définition autonomiste de la littérature" (ibid.): le fait littéraire francophone s'impose "comme une réalité construite", et est inséparable "d'une élaboration idéologique et politique" (p. 45) dont les contours nécessitent d'être tracés. Il invite dès lors à ne pas sousestimer les conditions matérielles qui permettent l'existence de collections et d'éditeurs francophones ou africains subventionnés tour à tour par l'État français ou par la Francophonie, et à tenter de concevoir la sociabilité des milieux intellectuels périphériques dans leur spécificité.

5 L'un des premiers reproches qui, dans le temps, furent adressés aux chercheurs abordant les littératures africaines par une méthode institutionnelle, était précisément celui de l'inadéquation de ce modèle, élaboré en Occident et pour l'Occident, appliqué à un corpus africain. Se greffant sur cette réflexion, Bernard Mouralis s'interroge sur la Pertinence de la notion de champ littéraire en littérature africaine et, en amont, sur l'exactitude de la perception diffuse d'une littérature africaine "objet global" analysable en tant que tel. Les questions posées sont loin d'être anodines: pour ce qui est du recours à la notion de champ littéraire, Mouralis se demande par exemple s'il 
faut considérer l'utilisation récente de cette notion par la critique "comme un nouveau moment dans le processus de diversification de l'objet que l'on se propose d'étudier ou, au contraire, comme une approche d'un autre type, dont le caractère pourrait même être de remettre en question la nature de l'objet" (p. 61), et cela vaut non seulement pour la production africaine, mais aussi pour le corpus de textes occidentaux sur l'Afrique (coloniaux et même postcoloniaux).

De la francophonie on passe, avec Jacqueline Bardolph, aux Conditions d'existence des champs littéraires d'Afrique anglophone: Bardolph les analyse avec les instruments de la sociologie, et soulève ensuite un certain nombre d'interrogations à propos des "retombées de ces conditions de production sur l'écriture même de la fiction" (p. 73). Elle se demande si l'on peut effectivement parler de champs littéraires à l'échelle d'un pays; s'il existe des lieux d'interaction entre la création et les conditions de productions locales; s'il existe une production portée par un public d'acheteurs (et non par les institutions éducatives) locaux. Après avoir présenté en exemple les littératures de l'Afrique du Sud, du Nigéria et du Zimbabwe, Jacqueline Bardolph démontre que les champs littéraires de l'Afrique anglophone dépassent nécessairement les frontières des nations singulières. On peut notamment distinguer trois espaces où les textes atteignent leur légitimité: celui qui s'est formé avec l'ancien pouvoir colonial; celui qui se définit par l'appartenance au Commonwealth; enfin celui du "dialogue international chaque jour plus clairement engagé avec les États-Unis". Quant à l'auteur de cet essai, elle souhaite vivement l'activation d'un nouveau champ à l'échelle continentale.

7 Jean Derive soulève une nouvelle question, tout à fait originale: il se demande si "l'oralité africaine se produit et se consomme selon des modalités susceptibles de donner lieu à des 'champs' comparables aux 'champs littéraires'“ décrits par Bourdieu, Dubois, etc. Ou encore, l'oralité africaine est en elle-même un champ littéraire (p. 87)? Par l'application de trois critères méthodologiques (historique, sociologique, textuel), l'auteur en arrive à la conclusion que, quoique "multiples et de visages divers", les littératures orales africaines font preuve d'une périodisation qui obéit à des principes qui ne sont pas étrangers à ceux des littératures issues des cultures écrites.

8 Un article de Janós Riesz, intitulé Littérature coloniale et littérature africaine: hypotexte et hypertexte ouvre une nouvelle section, intitulée "Limites et problématiques". Riesz défend la thèse selon laquelle la littérature coloniale française gagne à être lue en parallèle avec la littérature africaine de l'époque - et inversement, ces deux corpus étant envisagés sous les termes d'hypo- et d'hypertexte. La thèse et les exemples proposés se fondant en grande partie sur une expérience personnelle d'enseignement et de recherche, l'auteur conclut en livrant quelques conseils didactiques adressés aux chercheurs, aux professeurs qui donnent des cours de littérature coloniale et/ou africaine, aux auteurs de textes universitaires sur le même sujet, aux éditeurs susceptibles de rééditer certains ouvrages devenus introuvables.

9 La deuxième "problématique", introduite par Véronique Bonnet, concerne l'existence (les limites, la viabilité) du champ littéraire antillais. Une analyse de la nouvelle littérature de Guadeloupe et de Martinique (Glissant, Chamoiseau, Confiant, Maximin, Schwarz-Bart, Condé) lui permet de défendre l'idée d'un champ littéraire émergeant, en situation relativement autonome par rapport à la littérature nationale française (et à l'intérieur duquel la littérature créole serait un sous-champ), se définissant entre autres par un certain processus d'écriture fait de renvois intertextuels et paratextuels, qui reflètent "la genèse et le déploiement d'un véritable univers symbolique" (p. 147). 
“[...] il y a donc effet de champ sans que pour autant il n'y ait État-nation dans les îles des Petites Antilles francophones, ce qui amène à adapter les théories bourdieusiennes à cette réalité autre que celle décrite par le sociologue" (ibid.). Peter Hawkins, qui se questionne sur l'existence d'un champ littéraire mauricien, met en avant les mêmes précautions méthodologiques que Véronique Bonnet: il parait difficile d'appliquer les notions bourdieusiennes telles quelles à la réalité complexe et ambiguë de l'île Maurice. Mais les adaptations ne sont pas impossibles, si bien que l'auteur conclut en écrivant qu'il existe, effectivement, un champ littéraire mauricien, mais qu'il ne correspond pas à l'exemple du champ littéraire français de la deuxième moitié du XIX ${ }^{\mathrm{e}}$ siècle.

10 Anny-Claire Jaccard consacre sont intervention aux auteurs pieds-noirs: elle qualifie leur production d'"écriture" pieds-noirs, et non de littérature, celle-ci n'étant pas connue et reconnue en tant que telle, surtout en dehors d'un renvoi fort et constant à la France et à ses instances de consécration. Charles Djungu-Simba K. illustre une production éditoriale mal connue: la littérature de marché de Kinshasa, conçue dans le cadre plus vaste des lettres congolaises. Katharina Städtler conclut cette deuxième section du volume par un article intitulé La Négritude en France (1940-1950). À propos d'un champ littéraire colonisé en exil, qui se situe dans le cadre de l'historiographie littéraire du champ afro-francophone. La définition du titre s'explique par le fait que, pendant les années 1940-1950, presque toutes les instances du champ littéraire dont il est question se trouvent hors du continent africain, donc en exil, et sont dominées et monopolisées par le pouvoir colonial - d'où toute une série de stratégies d'émergence mises en œuvre par les auteurs africain, en vue d'une légitimation qui n'advient que lentement, au dépit de la fortune de la littérature coloniale française et du discours exotique romantique.

11 La troisième et dernière section du volume ("Pratiques, jeux et enjeux") abrite pour la plupart des études plus spécifiques, consacrées à des auteurs ou à des œuvres en particulier. La premier article fait partiellement exception (il sert en réalité de lien entre cette section et la précédente), dans la mesure où Florence Paravy y aborde le réseau thématique et symbolique de l'altérité comme enjeu du champ littéraire africain. Si l'on a si souvent parlé d'identité et d'altérité à propos des écrivains africains, c'est aussi en fonction de ce "double jeu" qu'il leur faut pratiquer en vue d'une légitimation: multiplier les signes renvoyant à une africanité plus ou moins sentie, par connivence avec le lecteur autochtone, et en même temps essayer de conquérir les institutions littéraires étrangères, pour accéder à une position reconnue.

En abordant La question du destinataire dans "Les Soleils des indépendances" d'Ahmadou Kourouma, Pierre Soubias va en réalité beaucoup plus loin: s'il rappelle le parcours de ce texte vers la reconnaissance, c'est afin de "dégager ce que cette histoire nous apprend sur l'état du champ littéraire africain pendant les années 1960" (p. 229), un champ qui n'a pas encore atteint un niveau de structuration très élevé, où la légitimation est attribuée, sinon directement par la France, du moins suivant des critères qui relèvent de son champ littéraire. Pierre Soubias reconnaît sans hésitations la révolution que constitue la force du style d'Ahmadou Kourouma, mais le mérite de son article réside dans le fait qu'il invite le lecteur à voir dans cet effort moins un désir gratuit de renouvellement esthétique, qu'"un écho des crises qui agitent en profondeur un certain champ de production culturelle" (p. 241).

Une étude de l'"affaire" Calixthe Beyala (la romancière camerounaise a été accusée de plagiat en 1996) permet à Madeleine Borgomano de se poser un certain nombre de 
questions sur le rapport entre ces polémiques et les caractéristiques propres au champ littéraire africain, l'“affaire" ayant mis en évidence "la zone de turbulences particulièrement violentes dans laquelle s'instaure ce champ" (p. 247).

Les trois essais suivants présentent une même structure logique et méthodologique de fond: Justin Kalulu Binsanwa étudie l'intertextualité dans l'œuvre de Valentin-Yves Mudinbé, et illustre son écartèlement entre deux cultures différentes, renvoyant respectivement à l'Afrique (au Congo) et à l'Europe; Marie-Françoise Bidault aborde l'itinéraire et la production de Manuel dos Santos Lima dans le cadre de la littérature angolaise; Sélom Komlan Gbanou situe l'œuvre de Sénouvo Zinzou au confluent des principales tendances de la littérature togolaise des dernières décennies. L'article final est signé par Anny Wynchank, qui étudie la transposition cinématographique que le Sénégalais Djibril Diop Mambéty a faite, dans le film Hyènes, de la pièce de Friedrich Dürrenmatt $L a$ Visite de la vieille dame, et montre de la sorte quelles transformations un même objet littéraire peut subir lorsqu'il est transposé non seulement d'un espace géographique et culturel à un autre, mais aussi d'un champ de production à un autre.

15 L'interrogation multiple dont se nourrit ce volume (et, en amont, le colloque qui est à sa source) a de nombreux mérites, et conduit à des résultats hautement appréciables. Le mérite, par exemple, d'intégrer véritablement le questionnement théorique et l'analyse des cas particuliers, ceux-ci revenant constamment sur les assises des premiers essais, sans les trahir, les délaisser ou les ajuster. Inversement - et ce n'est pas un paradoxe - les cas particuliers montrent la nécessité d'une redéfinition de la règle générale, en vue d'une adéquation, et donc d'une meilleure compréhension des spécificités propres aux champs littéraires africains. Autant dire que la théorie générale survit et se renforce, du moment que ses assises de base prouvent leur validité, et que les applications et les modifications particulières ne déstabilisent pas ses fondements. 